\title{
Motivational design techniques to support healthy eating habits at work
}

\author{
Robin De Croon \\ Department of Computer Science \\ $K U$ Leuven \\ Leuven, Belgium \\ robin.decroon@kuleuven.be
}

\author{
Francisco Gutiérrez \\ Department of Computer Science \\ $K U$ Leuven \\ Leuven, Belgium \\ francisco.gutierrez@kuleuven.be
}

\author{
Rachel Berry \\ Food Databanks National Capability \\ Quadram Institute Bioscience \\ Norwich Research Park, UK \\ rachel.berry@quadram.ac.uk
}

\author{
Siân Astley \\ EuroFIR AISBL \\ EuroFIR \\ Brussels, Belgium \\ sa@eurofir.org
}

\author{
Katrien Verbert, Vero Vanden Abeele \\ Department of Computer Science \\ KU Leuven \\ Leuven, Belgium \\ katrien.verbert@kuleuven.be
}

\begin{abstract}
This exploratory study provides a deeper understanding of employees when they are asked to use a personalized meal recommendation application in the workplace. Motivational design techniques were integrated into 25 alternative designs and evaluated. Our initial results show that participants appreciated the designs and highlighted the importance of personalized goals, the option to monitor eating habits, and the need for validated, personalized nutritional information.
\end{abstract}

Index Terms-PERSFO, food, nutrition, service providers, EIT Food, personalization, motivational design techniques

\section{INTRODUCTION AND MOTIVATION}

Although people are increasingly aware that eating healthily is important. In Europe, the prevalence of obesity tripled between 1975-2016 [1]. Recent research has focused on personalized approaches to promote healthy eating patterns [2], [3]. However, while studies indicate that personalized dietary recommendations are adhered to more readily, and for longer than generic advice [4], many people still struggle to achieve their dietary goals and to eat healthily.

Increasingly, daily food intakes occur in retail-controlled service environments, such as catering in the workplace or take-away food service outlets. Consequently, food service providers offer enormous potential to help employees achieve dietary targets, triggering behavior changes in a controlled environment through food choices. While consumption of foods from catering outlets and vending machines is commonplace, generally, these do not facilitate healthy eating. "Linking personalized dietary advice directly to food service providers could be an important step in helping consumers choose healthy food in and away from home." [5]

A vast body of literature exists on health behavior, behavior change, and motivational psychology [6]. However, translating

This work was undertaken within PERSFO Task A2002: $360^{\circ}$ Stakeholder analysis; Personalized and connected food service providers (PERSFO, Project ID 20291) received funding from EIT Food, the innovation community on Food of the European Institute of Innovation and Technology (EIT), a body of the EU, under Horizon 2020, the EU Framework Programme for Research and Innovation - www.persfo.eu these theoretical models and constructs into actionable, motivational app features is still an open research challenge [7]. In this exploratory study, based on [8], we aimed to evaluate how employees perceive motivational design techniques supporting food recommendations in a workplace context. The broader goal of this research study was to inform researchers and developers of food recommender applications about which motivational design techniques can promote increased adherence to healthy eating habits at work. Hence, this study provides implications for the design and application of motivational design techniques in food recommender application to support healthy eating habits at work.

\section{APPROACH}

Project goals that contextualize this study are described in [5]. Due to Belgian COVID19 public health measures, this study was performed online and employed a mixed-method approach; quantitative analysis of how employees and food experts rated motivational design techniques and qualitative analysis of the user centered design sessions. An overview of this exploratory study is shown in Fig. 1 and consisted of three focus groups, one co-design session, and one semi-structured interview with two dietitians. Ethical approval was granted by the Ethics Committee of KU Leuven (G 201912 1911).

We complimented the motivational design techniques used in De Croon et al. [8] with the nudging framework of Caraban et al. [9] to tailor the techniques to a professional work environment. Two lead researchers, in close collaboration with food and technical experts, designed a final set of 25 designs that were rated in three focus groups. A subset of these designs is illustrated in Fig. 2; all designs are available at https://www.eurofir.org/persfo/designs/.

Each focus group consisted of two parts:

part 1: Participants were asked to fill out the mobile device proficiency questionnaire [10] and the sport motivation scale [11], adapted for healthy eating. Participants were presented with 25 low-fidelity designs and asked to rate them on 


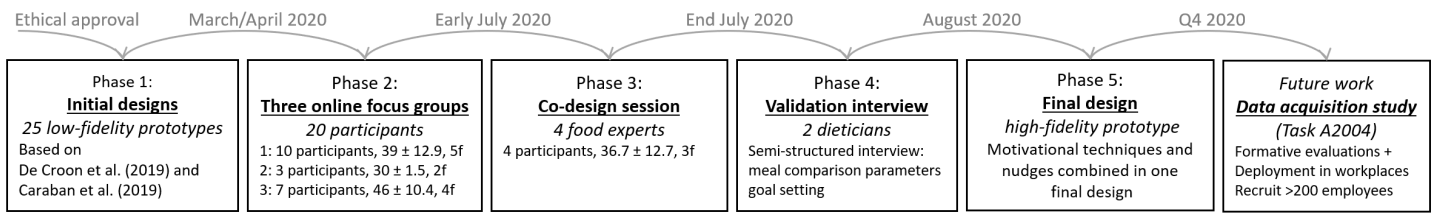

Fig. 1. Timeline that shows the studies and the participants. This paper considers phase 1-4. The final designs of Phase 5 will be presented on the poster.
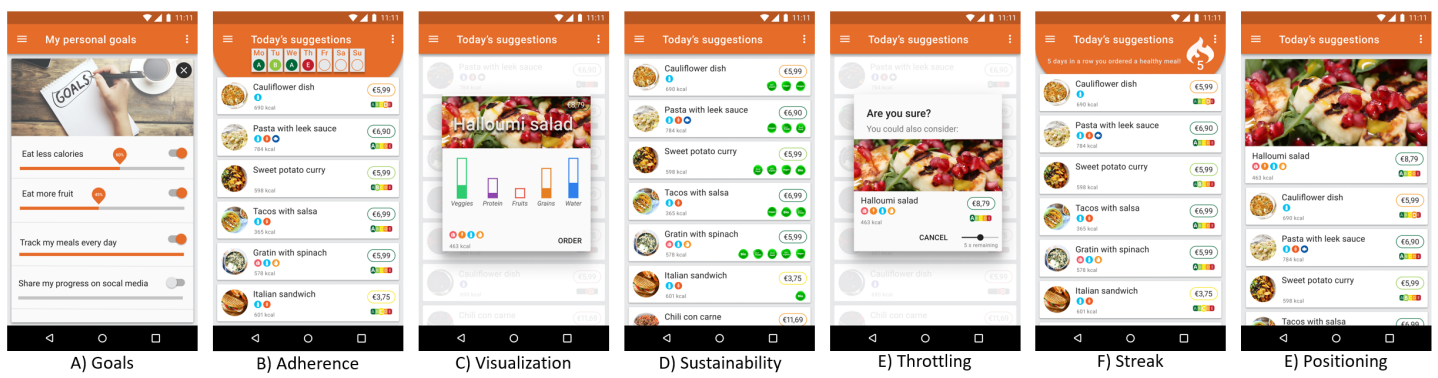

Fig. 2. Overview of seven out of 25 designs. All designs were rated on a seven-point Likert scale and discussed afterwards.

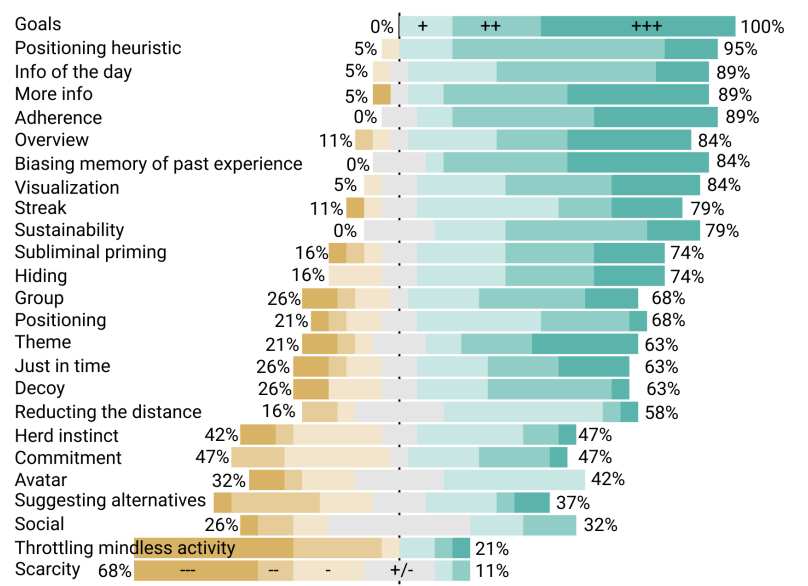

Fig. 3. Diverging bar chart visualizing how participants $(n=20)$ scored all 25 designs on a seven-point Likert scale. Sorted by participants' preference. The percentages indicated how many participants perceived a design as positive (right) and negative (left).

a seven-point Likert scale.

part 2: Participants were invited to discuss their attitudes to an mHealth app supporting healthy eating behavior and their evaluations of the different motivational designs.

\section{RESUlTS AND Discussion}

As illustrated in Fig. 3, participants perceived most designs favorably. To prioritize the designs, we define positive by a median score $\geq$ '++'. Within this definition, participants scored at least ten positive: goals, position heuristic, info of the day, more info, adherence, overview, biasing memory of past experience, visualization, sustainability, and theme.

Overall best-perceived was the goals (Fig. 2A) design, which allowed employees to set their personalized goals; this was confirmed in the follow-up design sessions. Experts stressed that goals should be user configurable and not linked directly to a specific diet. Instead, goals should focus on reducing unhealthy eating more than increasing healthy eating or reducing total eating [12], e.g., eating less saturated fats, reducing alcohol intake, etc.

As shown by higher scores for more info, adherence (Fig. 2B), overview, visualization (Fig. 2C), etc., a second recurring need was feedback. Similar to [8], participants indicated they intended to adhere to their goals, but often lacked tools to monitor progress "Would be great to also have an overview of your goal, to see how well you're doing."

The higher ranking of info of the day, more info, sustainability (Fig. 2D) also show that employees need understandable and validated information: "It is good to know what you are eating." Most information that is available online is of questionable quality [13], making it harder for motivated individuals to find validated information. Moreover, it is not personalized: "Talking about how healthy an apple is, is not useful when you are allergic to apples."

Finally, similar to [8], features that should be avoided in a food recommender app are those taking away control from the individual, e.g., throttling mindless activity (Fig. 2E), features that lead to unprofessional/childish look and feel, e.g., inappropriate avatar for audience, and features that might imply pressure on the employee.

\section{CONCLUSION AND Future WORK}

Building on previous work, our results show that a personalized combination of motivational design techniques are needed. Three design requirements are that (1) employees should be able to set their goals linked to mindful eating, (2) employees should be able to analyze progress towards their goals and eating habits, and (3) validated, personalized information should be available. The app is under development and will be available for employees from participating companies, with the initial aim to measure the acceptance and collect data for a larger, future validation study. 


\section{REFERENCES}

[1] World Health Organization, "Obesity and overweight," https://www.who.int/news-room/fact-sheets/detail/obesity-andoverweight, Apr. 2020, accessed: 2020-8-24.

[2] D. Toro-Martín, B. J. Arsenault, J.-P. Després, M.-C. Vohl et al., "Precision nutrition: a review of personalized nutritional approaches for the prevention and management of metabolic syndrome," Nutrients, vol. 9 , no. 8, p. $913,2017$.

[3] D. S. Lizano, J. Goossens, S. Astley, R. Berry, and P. Finglas, "Quisper and development of a personalised nutrition community," AgroFOOD Industry $\mathrm{Hi}$ Tech, vol. 31, no. 2, pp. 36-38, 2020.

[4] A. Ronteltap, H. van Trijp, A. Berezowska, and J. Goossens, "Nutrigenomics-based personalised nutritional advice: in search of a business model?" Genes \& nutrition, vol. 8, no. 2, pp. 153-163, 2013.

[5] J. Goossens, S. Astley, G. Mathijssen, H. Simillion, P. Finglas, and D. S. Lizano, "Introducing personalised nutrition services in a behavioural motivation concept for connected food service environments," AgroFOOD Industry Hi Tech, vol. 31, no. 2, pp. 42-45, 2020.

[6] R. De Croon, D. Wildemeersch, J. Wille, K. Verbert, and V. V. Abeele, "Gamification and serious games in a healthcare informatics context," in 2018 IEEE International Conference on Healthcare Informatics (ICHI). IEEE, 2018, pp. 53-63.

[7] J. Geuens, L. Geurts, K. Gerling, R. De Croon, and V. V. Abeele, "A dyad of lenses for the motivational design of mhealth: Bridging the gap between health theory and app design," in 2019 IEEE International Conference on Healthcare Informatics (ICHI). IEEE, 2019, pp. 1-12.
[8] R. De Croon, A. M. Bezabih, J. Geuens, D. Wildemeersch, D. Oeyen, $\mathrm{K}$. Verbert, and V. V. Abeele, "Motivational design techniques to increase adherence to a telemonitoring therapy a study with adolescent pectus patients," in 2019 IEEE International Conference on Healthcare Informatics (ICHI). IEEE, 2019, pp. 1-12.

[9] A. Caraban, E. Karapanos, D. Gonçalves, and P. Campos, "23 ways to nudge: A review of technology-mediated nudging in human-computer interaction," in Proceedings of the 2019 CHI Conference on Human Factors in Computing Systems, 2019, pp. 1-15.

[10] N. A. Roque and W. R. Boot, "A new tool for assessing mobile device proficiency in older adults: the mobile device proficiency questionnaire," Journal of Applied Gerontology, vol. 37, no. 2, pp. 131-156, 2018.

[11] L. G. Pelletier, M. A. Rocchi, R. J. Vallerand, E. L. Deci, and R. M. Ryan, "Validation of the revised sport motivation scale (sms-ii)," Psychology of sport and exercise, vol. 14, no. 3, pp. 329-341, 2013.

[12] R. Cadario and P. Chandon, "Which healthy eating nudges work best? a meta-analysis of field experiments," Marketing Science, vol. 39, no. 3, pp. 465-486, 2020.

[13] L. Daraz, A. S. Morrow, O. J. Ponce, B. Beuschel, M. H. Farah, A. Katabi, M. Alsawas, A. M. Majzoub, R. Benkhadra, M. O. Seisa et al., "Can patients trust online health information? a meta-narrative systematic review addressing the quality of health information on the internet," Journal of general internal medicine, pp. 1-8, 2019. 